\title{
Original Study \\ Current Prescribing Practices for Skin and Soft Tissue Infections in Nursing Homes
}

\author{
Diana Feldstein MD ${ }^{\mathrm{a}, *}$, Philip D. Sloane MD, MPH ${ }^{\mathrm{b}, \mathrm{c}}$, David Weber MD, MPH ${ }^{\mathrm{d}}$, \\ Kimberly Ward $\mathrm{BA}^{\mathrm{c}}$, David Reed $\mathrm{PhD}^{\mathrm{c}}$, Sheryl Zimmerman $\mathrm{PhD}^{\mathrm{c}, \mathrm{e}}$ \\ a Division of Geriatric Medicine, Center for Aging and Health, University of North Carolina, Chapel Hill, NC \\ ${ }^{\mathrm{b}}$ Department of Family Medicine, University of North Carolina, Chapel Hill, NC \\ ${ }^{\mathrm{c}}$ Cecil G. Sheps Center for Health Services Research, University of North Carolina, Chapel Hill, NC \\ ${ }^{\mathrm{d}}$ Division of Infectious Diseases, University of North Carolina, Chapel Hill, NC \\ e School of Social Work, University of North Carolina, Chapel Hill, NC
}

Keywords:

Skin infection

nursing home

antibiotic prescribing

\begin{abstract}
A B S T R A C T
Objectives: Antibiotic stewardship has been called for across all sites of health care, including nursing homes (NHs). Skin and soft tissue infections (SSTIs) are the third most common indication for antibiotics in the $\mathrm{NH}$, and so should be a focus of stewardship. This study audited medical records to identify signs and symptoms of SSTIs treated with antibiotics in relation to the McGeer criteria for surveillance, the Loeb minimum criteria for antibiotic initiation, and prescribing recommendations of the Infectious Disease Society of America.

Design: Cross-sectional study.

Setting: Thirty-one NHs in Southeastern United States.

Measurements: Chart data from a random sample of 161 antibiotic prescriptions for SSTIs were abstracted. To meet the McGeer criteria, pus was present at a suspected SSTI site, or at least four of the following findings were documented as present at the site: new or worsening warmth, redness, swelling, tenderness, serous drainage, or a constitutional finding. The Loeb minimum criteria for initiating antibiotics included findings of new or increasing purulent drainage at a suspected SSTI site or at least two of the following findings: fever or new or worsening redness, tenderness, warmth, or swelling at the suspected site. Audits also collected the name, route, and duration of the associated antibiotic. Analyses calculated the types of diagnoses and evaluated associations between published criteria and prescribing. Results: Cellulitis, skin/soft tissue injury with infection, and abscess were diagnosed in $37 \%(\mathrm{~N}=59), 18 \%$ $(\mathrm{N}=29)$, and $16 \%(\mathrm{~N}=26)$ of cases, respectively; $27 \%(\mathrm{~N}=43)$ had less specific diagnoses. The McGeer criteria were met in $25 \%(\mathrm{~N}=40)$, and the Loeb minimum criteria were met in $48 \%(\mathrm{~N}=77)$ of cases. Doxycycline was the most frequently prescribed antibiotic. The mean treatment length was 9.6 days (standard deviation, 5.6), and the median length of treatment was 8.5 days (range, 3-45).

Conclusion: SSTIs are not routinely diagnosed or treated according to recommended standards of care, and prescriptions for systemic antibiotics appear to be frequently initiated without regard to recommended definitions of infection or therapies for the associated diagnoses. These findings indicate that SSTIs present various opportunities to improve antibiotic stewardship.
\end{abstract}

\footnotetext{
The authors declare no conflicts of interest.

Funding sources: Grant \#R18 HS022846-01 from the U.S. Agency for HealthCare Research and Quality and the John A. Hartford Foundation Center of Excellence in Geriatric Medicine and Training Renewal "Carolina Center of Excellence in Geriatric Medicine" grant.

The authors express their appreciation to the staff of the nursing homes that participated in the project.
}

Portions of this article were presented at the annual meeting of the American Geriatrics Society, Long Beach, California, May 19, 2016.

* Address correspondence to Diana Feldstein, MD, Division of Geriatrics, University of North Carolina at Chapel Hill, 5003 Old Clinic, CB 7550, Chapel Hill, NC 27599.

E-mail address: diana_feldstein@med.unc.edu (D. Feldstein). 
As many as one-third of nursing home $(\mathrm{NH})$ residents carry one or more multidrug-resistant organisms (MDROs), ${ }_{1}^{1}$ with colonization of new residents tending to occur shortly following admission. ${ }^{2}$ To limit further resistance, the U.S. Centers for Disease Control and Prevention recently encouraged all NHs to develop antibiotic stewardship programs as a new standard in care. ${ }^{3}$ In addition, the U.S. Centers for Medicare and Medicaid Services is considering mandating that all NHs have antibiotic stewardship programs. ${ }^{4}$ When determining the focus of these programs, it is important to recognize that skin and soft tissue infections (SSTIs) are the third most common indication for antibiotics in $\mathrm{NHs}^{5,6}$ and therefore an important target for antibiotic stewardship.

Antibiotic stewardship for skin infections in NHs presents unique challenges. $\mathrm{NH}$ residents have a particularly high prevalence of comorbid health illnesses, such as peripheral vascular disease, venous stasis disease, and pressure ulcers, ${ }^{7}$ which contribute to chronic skin changes. These conditions can obscure the distinction between acute changes and chronic findings. Also, many $\mathrm{NH}$ residents have chronic wounds that are colonized with bacteria $^{8}$ and potentially harbor MDROs. In addition, dementia is common, which hinders diagnoses by limiting information gathering regarding symptoms. Finally, NH residents live in a communal environment and receive assistance from caregivers who move from resident to resident, often using shared medical supplies, which facilitates the spread of infectious organisms. $^{9}$

A number of criteria have been promulgated by experts to identify when antibiotics are appropriate for SSTIs in NHs. Loeb and colleagues developed minimum consensus criteria for antibiotic initiation in commonly encountered $\mathrm{NH}$ bacterial infections. ${ }^{10}$ Although there is evidence that adherence to the Loeb criteria is not associated with antibiotic prescribing decisions, ${ }^{11}$ these remain the most popular criteria for prospective use in determining antibiotic initiation. Another set of consensus criteria, the McGeer criteria, were introduced in 1991 to direct surveillance of common infections in $\mathrm{NHs}^{12}$ and were revised in $2012 .^{13}$ An additional set of guidelines for antibiotic use in SSTIs, those of the Infectious Disease Society of America (IDSA), was developed in 2014 for SSTIs in community-dwelling patients. ${ }^{14}$ Whether and to what extent these criteria can or do influence decision making in NHs is unknown.

Given the importance of SSTIs in NH practice and the mounting pressure for antibiotic stewardship, surprisingly little data exist regarding how presumed SSTIs are actually diagnosed and managed in $\mathrm{NH}$ practice. To address this knowledge gap, we studied 161 antibiotic prescriptions for SSTIs in $30 \mathrm{NHs}$ in North Carolina. We audited medical records for documentation of signs and symptoms related to an SSTI according to the definitions in the recently updated McGeer criteria and Loeb minimum criteria. We also present the treatment courses in relation to the IDSA guidelines. The results are highly informative to guide antibiotic stewardship efforts in NHs.

\section{Methods}

As part of a dissemination research study of infection management and antibiotic stewardship in NHs, we enrolled 31 NHs in North Carolina and, within each, audited a random sample of antibiotic prescriptions for SSTIs. We collected chart documentation regarding any signs and symptoms of a possible SSTI as articulated in the modified McGeer criteria and the Loeb minimum criteria and related these clinical data to the IDSA recommendations for empirical antibiotic therapy.

Eligible NHs were affiliated with either a regional $\mathrm{NH}$ chain or a regional long-term care medical practice group. A total of $35 \mathrm{NHs}$ were approached for participation. Four refused, and the remaining 31 (89\%) enrolled in the study. Participating NHs provided monthly lists of all antibiotic prescriptions and the diagnoses as reported for each by the NH between September 2014 and March 2015; during this period,
$30 \mathrm{NHs}$ (97\%) reported antibiotic prescriptions for one or more SSTI. A team of geriatricians and research staff visited each $\mathrm{NH}$ and conducted chart audits of up to eight prescriptions for SSTIs from the month prior to the audit, yielding a sample of 161 cases of treated SSTIs from 30 NHs.

The medical and nursing records of the selected charts of residents who received an antibiotic for an SSTI were systematically audited for documentation of elements of the modified McGeer criteria and the Loeb minimum criteria recorded in the 48 hours prior to the initiation of the antibiotic. The McGeer criteria were met if pus was present at a suspected SSTI site or if at least four of the following were present at the site: new or worsening warmth, redness, swelling, tenderness, serous drainage, or a constitutional finding. According to these criteria, constitutional findings included fever, leukocytosis, acute change in mental status and functional decline; however, change in mental status and function were excluded from data collection and analysis because no $\mathrm{NH}$ documented them in the manner specified by the McGeer criteria. ${ }^{13}$ We recorded fever as a single oral temperature $>37.8^{\circ} \mathrm{C}\left(100^{\circ} \mathrm{F}\right)$ or $>37.2^{\circ} \mathrm{C}\left(99^{\circ} \mathrm{F}\right) 48$ hours before a prescription, similar to but not consistent with the revised McGeer criteria (which insist on two oral temperatures for the lower threshold). ${ }^{13}$ Documentation of the other constitutional finding, leukocytosis ( $>14,000$ leukocytes $/ \mathrm{mm}^{3}$ or $>6 \%$ bands or $>1,500$ bands $/ \mathrm{mm}^{3}$ ) was collected within 72 hours of a prescription. Additional data including the source, type, and results of SSTI cultures were gathered. Audits also recorded the frequency of emergency department visits, hospitalizations, and deaths in the 7 days following an antibiotic prescription.

The Loeb minimum criteria share many of the clinical findings of the McGeer criteria. They recommend antibiotic initiation for an SSTI if new or increasing purulent drainage is noted at a wound, skin, or soft-tissue site-or at least two of the following findings: fever (temperature $>37.9^{\circ} \mathrm{C}\left[100^{\circ} \mathrm{F}\right]$ or an increase of $1.5^{\circ} \mathrm{C}\left[2.4^{\circ} \mathrm{F}\right]$ above baseline temperatures taken at any site) or new or worsening redness, tenderness, warmth, or swelling at the site. ${ }^{10}$ Our audits collected all of these data.

Diagnoses were recorded verbatim from notes written by medical providers (physicians, nurse practitioners, or physician assistants) or, if no diagnosis was present in a provider note, it was obtained from the nursing notes. Diagnoses were then aggregated into up to three ICD-9 (International Classification of Diseases, 9th revision) categories by project staff, under the direction of two geriatricians (D.F. and P.D.S.). Cases with nonspecific diagnoses such as "skin infection" were coded as ICD-9 709.9 "unspecified disorder of skin and subcutaneous tissue." 15

Analyses calculated the percent of diagnostic types and evaluated associations between published criteria and antibiotic prescribing for the three most common ICD-9 categories; that is, for each of the three diagnostic categories, the proportion of prescriptions that met the modified McGeer criteria and the Loeb minimum criteria was calculated. Analyses adjusted for differences in the method of measuring temperature by subtracting $0.75^{\circ} \mathrm{F}$ from rectal and tympanic readings and adding $0.75^{\circ} \mathrm{F}$ to axillary readings, to obtain an oral temperature equivalent. ${ }^{16}$ In addition, empirical antibiotic choices were compared to those recommended by the IDSA for a specific diagnosis. The study was approved by the University of North Carolina Institutional Review Board.

\section{Results}

The mean bed size of the 31 participating NHs was 113 (standard deviation [SD], 50; range, 40-217); the mean occupancy was $87 \%$; $74 \%$ were part of a chain, and $81 \%$ were for profit. The mean quality rating from Nursing Home Compare was 3.3 of a maximum score of $5 .^{17}$ None of these values was significantly different from those of NHs across the nation (all $P>.05$ ). In the past 5 years, the average study $\mathrm{NH}$ had two 
different medical directors (range, 1-3), three nursing directors (range, 1-12), and two infection control nurses (range, $0-12$ ). The average $\mathrm{NH}$ medical director cared for $81 \%$ of all NH residents (range, 15\%-100\%). The average infection control nurse spent 7.9 (SD, 6.1) hours a week devoted to infection control. The average NH had 32 (SD, 14) licensed nurses (RN/LPN) on staff. Fifty-two percent of NHs had a nursing wound specialist, and $58 \%$ had at least one physician trained in wound care. Study NHs had an average of $27 \%$ of all residents in private rooms.

The $\mathrm{NH}$ residents whose records were audited averaged 82 years old. Thirty percent were male, 32\% were African American, and 5\% were on hospice care. One resident had an order to withhold antibiotics.

A total of 161 antibiotic prescriptions for SSTIs were audited across the 30 study NHs. Seventy percent $(\mathrm{N}=112)$ of the abstracted SSTI prescriptions were initiated at the $\mathrm{NH}$; the remaining $30 \%(\mathrm{~N}=49)$ were prescribed at an emergency department, during a hospitalization, at a physician's office, or at an unknown setting. The proportion of diagnoses and associated findings for SSTI prescriptions is displayed in Figure 1. The three most frequent diagnoses were cellulitis $(\mathrm{N}=59$, $37 \%$ ), skin/soft tissue injury with infection ( $\mathrm{N}=29,18 \%$ ), and abscess $(\mathrm{N}=26,16 \%)$. Eight percent $(\mathrm{N}=13)$ of prescriptions were given for wounds with characteristics of pressure ulcers (data not shown), but the specific diagnosis of pressure ulcer infection was documented in only $2 \%(\mathrm{~N}=4)$. Wounds of any kind were described in $27 \%(\mathrm{~N}=44)$ of SSTI prescriptions (data not shown). Finally, $27 \%(\mathrm{~N}=43)$ of antibiotics were prescribed for "unspecified disorder of skin and subcutaneous tissue" including diagnoses such as "contusion," "bacterial infection," "MRSA," or "ankle infection."

Infection quality assurance requires documentation. ${ }^{13}$ However, the majority of treated cases did not have documentation that either supported or invalidated the associated diagnoses. As shown in the bottom row of Table 1 , sufficient documentation was present to meet the modified McGeer criteria for an SSTI in $25 \%(\mathrm{~N}=40)$ of the 161 study prescriptions. Sixteen percent $(\mathrm{N}=25)$ of all prescriptions had no modified McGeer criteria documented (data not shown). The Loeb minimum criteria were satisfied in $48 \%(\mathrm{~N}=77)$ of prescriptions.

Audits found the presence of pus documented at the site of the suspected SSTI in $18 \%(\mathrm{~N}=30)$ of prescriptions. Redness was the most frequently documented sign in cases of treated cellulitis ( $54 \%, \mathrm{~N}=32)$ and treated cases overall $(42 \%, \mathrm{~N}=67)$. The presence or absence of redness was not documented at all in $53 \%$ of treated cases. Documentation of the presence or absence of the remaining McGeer criteria-swelling, tenderness, or serous drainage, was absent in $71 \%$ $(\mathrm{N}=115), 63 \%(\mathrm{~N}=102)$, and $70 \%(\mathrm{~N}=113)$ of cases, respectively. Almost one-third (32\%, $\mathrm{N}=52$ ) of SSTI prescriptions were initiated without any temperature documented within 48 hours of treatment initiation.

Laboratory data (including a culture from a suspected SSTI site or a white blood cell count) were infrequently documented. Leukocytosis, a constitutional finding of the McGeer criteria, was noted in only one prescription, and a white blood cell count within 72 hours of treatment initiation was available in only $7 \%(\mathrm{~N}=12)$ of audited charts. Cultures from sites of a suspected SSTI were obtained for $9 \%(\mathrm{~N}=14)$ of the 161 prescriptions; of these, eight were documented to be obtained from wounds, and six did not have a source documented. The technique used to capture samples (eg, Levine technique, needle aspiration, or surface swab) was never documented. Of the 14 cultures, half revealed methicillin-resistant Staphylococcus aureus, three grew Klebsiella species, two resulted in Pseudomonas species, 2 had polymicrobial results, and six were associated with antibiotic regimen changes.

The mean duration for any prescription was 9.6 days (SD, 5.9), and the median number of treatment days was 8.5 (range, 3-45; see Table 2). Doxycycline was the most frequently prescribed antibiotic

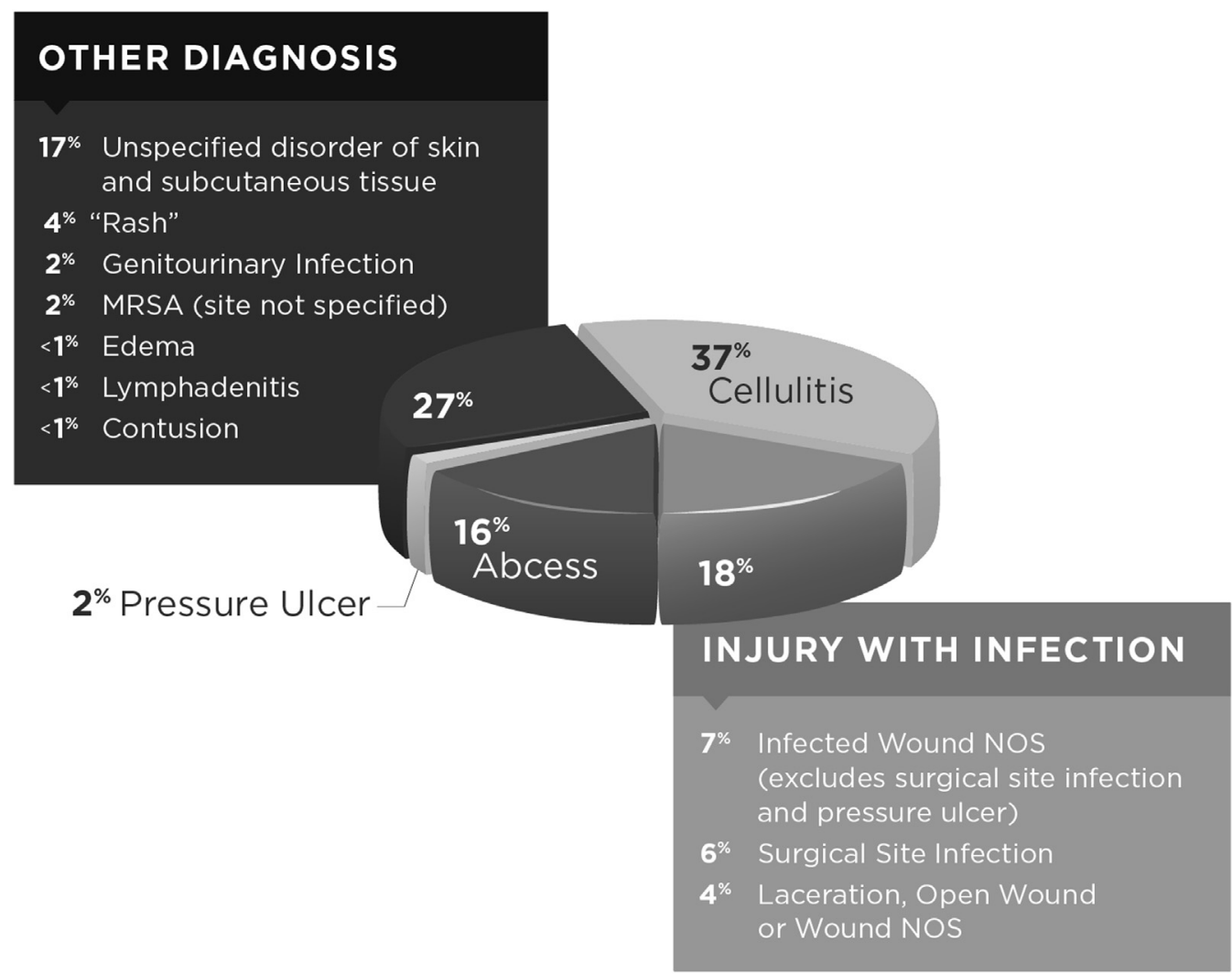

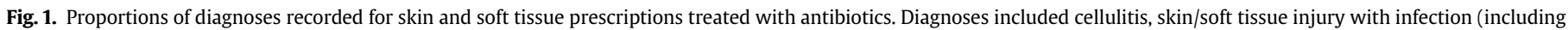

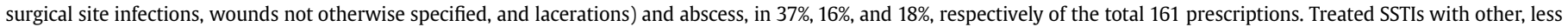
specific, indications constituted $27 \%$ of total prescriptions. 
Table 1

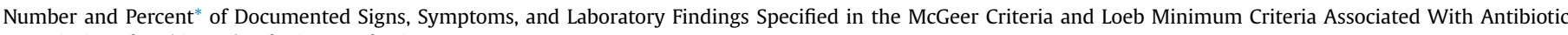
Prescriptions for Skin and Soft Tissue Infections

\begin{tabular}{|c|c|c|c|c|}
\hline \multirow[t]{2}{*}{ Signs, Symptoms, and Laboratory Findings } & \multicolumn{3}{|l|}{ Type of SSTI } & \multirow{2}{*}{$\begin{array}{l}\text { Total Sampled } \\
\text { Prescriptions, } \\
\mathrm{N}=161\end{array}$} \\
\hline & $\begin{array}{l}\text { Cellulitis, } \\
\mathrm{N}=59,37 \% \\
\text { of Prescriptions }\end{array}$ & $\begin{array}{l}\text { Skin/Soft Tissue } \\
\text { Injury with Infection }{ }^{\dagger}, \\
\mathrm{N}=29,18 \% \text { of } \\
\text { Prescriptions }\end{array}$ & $\begin{array}{l}\text { Abscess, } \\
\mathrm{N}=26,16 \% \\
\text { of Prescriptions }\end{array}$ & \\
\hline \multicolumn{5}{|l|}{$\mathrm{Pus}^{\dagger, 8}$} \\
\hline Yes & $3(5 \%)$ & $10(34 \%)$ & $8(31 \%)$ & 30 (19\%) \\
\hline No & $4(7 \%)$ & $2(7 \%)$ & $3(12 \%)$ & $14(9 \%)$ \\
\hline Not documented & $52(88 \%)$ & 17 (59\%) & $15(57 \%)$ & 117 (73\%) \\
\hline \multicolumn{5}{|l|}{ Heat/warmth } \\
\hline Yes & $16(27 \%)$ & $8(28 \%)$ & $3(12 \%)$ & 31 (19\%) \\
\hline No & $5(8 \%)$ & $3(10 \%)$ & $1(4 \%)$ & $14(9 \%)$ \\
\hline Not documented & $38(64 \%)$ & $18(62 \%)$ & $22(85 \%)$ & $116(72 \%)$ \\
\hline \multicolumn{5}{|l|}{ Redness $^{\dagger, \delta}$} \\
\hline Yes & $32(54 \%)$ & $10(35 \%)$ & $9(35 \%)$ & 67 (42\%) \\
\hline No & $3(5 \%)$ & $3(10 \%)$ & $1(4 \%)$ & $9(6 \%)$ \\
\hline Not documented & $24(41 \%)$ & $16(55 \%)$ & $16(62 \%)$ & $85(53 \%)$ \\
\hline \multicolumn{5}{|l|}{ Swelling ${ }^{\ddagger \S}$} \\
\hline Yes & $22(37 \%)$ & $3(10 \%)$ & $5(19 \%)$ & $34(21 \%)$ \\
\hline No & $3(5 \%)$ & $4(14 \%)$ & $1(4 \%)$ & $12(8 \%)$ \\
\hline Not documented & $34(58 \%)$ & $22(76 \%)$ & $20(77 \%)$ & $115(71 \%)$ \\
\hline \multicolumn{5}{|l|}{ Tenderness ${ }^{\ddagger \S}$} \\
\hline Yes & $17(29 \%)$ & 7 (24\%) & $9(35 \%)$ & $45(28 \%)$ \\
\hline No & $6(10 \%)$ & $3(10 \%)$ & $1(4 \%)$ & $14(9 \%)$ \\
\hline Not documented & $36(61 \%)$ & $19(66 \%)$ & $16(61 \%)$ & $102(63 \%)$ \\
\hline \multicolumn{5}{|l|}{ Serous drainage } \\
\hline Yes & $5(8 \%)$ & $7(24 \%)$ & $3(12 \%)$ & $23(14 \%)$ \\
\hline No & $6(10 \%)$ & $4(14 \%)$ & $8(31 \%)$ & $25(16 \%)$ \\
\hline Not documented & $48(81 \%)$ & $18(62 \%)$ & $15(58 \%)$ & $113(70 \%)$ \\
\hline \multicolumn{5}{|l|}{ Fever } \\
\hline 1 temperature $\geq 100.0^{\circ} \mathrm{F}^{\dagger, \S}$ & $0(0 \%)$ & $1(3 \%)$ & $1(4 \%)$ & $3(2 \%)$ \\
\hline$\geq 2$ temperatures $>99^{\circ} \mathrm{F}^{\ddagger}$ & $1(2 \%)$ & $2(7 \%)$ & $1(4 \%)$ & $5(3 \%)$ \\
\hline $\bar{N}$ o temperature documented & $19(32 \%)$ & $8(28 \%)$ & $9(35 \%)$ & $52(32 \%)$ \\
\hline \multicolumn{5}{|l|}{ WBC count } \\
\hline WBC count available & $5(8 \%)$ & $3(10 \%)$ & $1(4 \%)$ & $12(7 \%)$ \\
\hline Leukocytosis & $1(2 \%)$ & $0(0 \%)$ & $0(0 \%)$ & $1(1 \%)$ \\
\hline Met modified McGeer criteria (pus or $\geq 4$ of 7 indicators ${ }^{\ddagger}$ ) & $8(14 \%)$ & $12(41 \%)$ & $10(38 \%)$ & $40(25 \%)$ \\
\hline Met the Loeb minimum criteria (pus or $\geq 2$ of 5 indicators $^{5}$ ) & $32(54 \%)$ & $14(48 \%)$ & $14(54 \%)$ & $77(48 \%)$ \\
\hline
\end{tabular}

SSTI, skin and soft tissue infection; WBC, white blood cell.

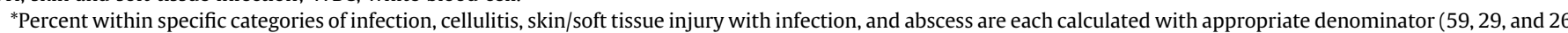

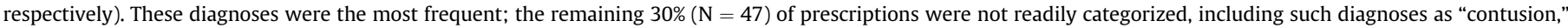

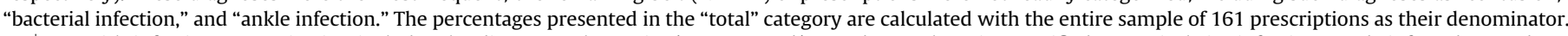

'SSTI with infection categorization includes the diagnoses "laceration/open wound/wound not otherwise specified," "surgical site infection," and "infected wound not otherwise specified (excludes pressure ulcer)."

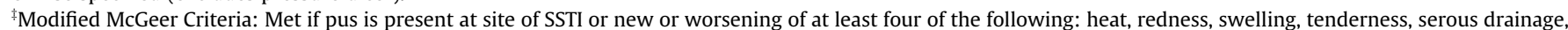
fever $\left(>100.0^{\circ} \mathrm{F}, \geq 2\right.$ temperatures greater than $99^{\circ} \mathrm{F}$ ) or leukocytosis $\left(\geq 14,000\right.$ leukocytes $/ \mathrm{mm}^{3}$ or $\geq 6 \%$ bands or $\geq 1,500$ bands $/ \mathrm{mm}^{3}$ ) present. ${ }^{13}$

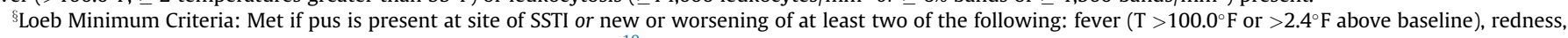
tenderness, warmth, new or increased swelling at the suspected site. ${ }^{10}$

regardless of the type of infection. Intravenous vancomycin was given in $5 \%(\mathrm{~N}=8)$ of all SSTIs. The initial antibiotic was changed or stopped in $13 \%(\mathrm{~N}=21)$ of the prescriptions, and an additional antibiotic was added in $6 \%(\mathrm{~N}=10)$.

\section{Discussion}

Twenty years ago, the Society for Healthcare Epidemiology of America reported inappropriately high antibiotic prescribing in NHs. ${ }^{18}$ The findings presented in this article of $161 \mathrm{NH}$ cases for which antibiotics were prescribed for SSTIs suggest that practices have not dramatically improved in the interim, despite calls for more judicious antibiotic prescribing and the development of new resources for clinicians. We found that few cases met the surveillance requirements of the modified McGeer criteria, and approximately half met the less stringent Loeb minimum criteria. The treatment courses often did not adhere to guidelines for given diagnoses and were frequently prolonged. Thus, treatment of presumed SSTIs in NHs offers considerable opportunity to improve antibiotic stewardship.
Cellulitis was the most commonly cited indication for SSTI treatment in our sample, similar to other NH studies. ${ }^{5}$ However, consistent with the overall results, cellulitis was diagnosed largely independently of the modified McGeer criteria definitions for an SSTI, as only $14 \%$ of cases met the surveillance definition. Prescribing was more in line with the Loeb criteria (54\%), however. The marked difference between compliance with the McGeer and Loeb criteria likely reflects the inherent challenges of using infection surveillance definitions to guide treatment decisions. For cellulitis, for example, the IDSA's clinical definition includes erythema, warmth and swelling, ${ }^{14}$ which together were documented as present in only $12 \%$ of treated cases. Redness was documented in $54 \%$ of prescriptions for cellulitis and was the only documented finding in $8 \%$ of treated cellulitis cases. Nevertheless, redness is also common in noninfectious, chronic skin changes such as stasis dermatitis, peripheral vascular disease, and venous insufficiency. Although antibiotics are not indicated for these and other noninfectious causes of erythema, our data suggest that the readily apparent finding of redness may influence potentially inappropriate prescribing. 
Table 2

Antibiotics Prescribed for Specific Diagnoses and of Total Sampled Prescriptions and Total Duration of Antibiotic Therapy

\begin{tabular}{|c|c|c|c|c|}
\hline \multirow[t]{2}{*}{ Antibiotics } & \multicolumn{3}{|l|}{ Type of SSTI } & \multirow{2}{*}{$\begin{array}{l}\text { Total Sampled } \\
\text { Prescriptions } \\
(\mathrm{N}=161)\end{array}$} \\
\hline & $\begin{array}{l}\text { Cellulitis } \\
(\mathrm{N}=59,37 \% \\
\text { of Prescriptions) }\end{array}$ & $\begin{array}{l}\text { SST Injury with } \\
\text { Infection* }(\mathrm{N}=29,18 \% \\
\text { of Prescriptions) }\end{array}$ & $\begin{array}{l}\text { Abscess, } \\
(\mathrm{N}=26,16 \% \\
\text { of Prescriptions) }\end{array}$ & \\
\hline Doxycycline & $17(29 \%)$ & $8(28 \%)$ & $14(54 \%)$ & $52(32 \%)$ \\
\hline Cephalexin & $14(24 \%)$ & $6(21 \%)$ & $6(23 \%)$ & $35(22 \%)$ \\
\hline Trimethoprim/sulfamethoxazole & $9(15 \%)$ & $6(21 \%)$ & $4(15 \%)$ & $23(14 \%)$ \\
\hline Clindamycin & $7(12 \%)$ & $1(3 \%)$ & $0(0 \%)$ & $11(7 \%)$ \\
\hline Amoxicillin-clavulanate & $4(7 \%)$ & $3(10 \%)$ & $0(0 \%)$ & $9(6 \%)$ \\
\hline Other antibiotics & $8(13 \%)$ & $5(17 \%)$ & $2(8 \%)$ & $31(19 \%)$ \\
\hline Mean number of days of treatment (SD) & $9.3(5.1)$ & $10.0(7.6)$ & $9.1(2.4)$ & $9.6(5.9)$ \\
\hline Median number of days of treatment (range) & $10(5-42)$ & $7(3-42)$ & $10(5-14)$ & $8.5(3-45)$ \\
\hline
\end{tabular}

SD, standard deviation; SSTI, soft tissue injury.

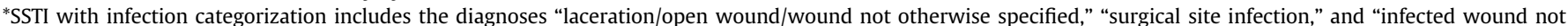
otherwise specified (excludes pressure ulcer)."

Regarding the lack of adherence to diagnostic guidelines, it is possible that the absence of documentation does not equate to the absence of clinical signs or symptoms, and so may not reflect lack of guideline adherence. However, given the increasing burden of MDROs and calls for antibiotic stewardship, better documentation is needed to provide reasonable justification for antibiotic use. After all, guidelines exist expressly to promote accurate diagnoses as justification for antibiotic use.

Our results indicate considerable nonadherence to guidelines regarding the type, route, and duration of antibiotic therapy. Cellulitis was treated most often with doxycycline rather than the recommended first-line penicillin-based therapies, ${ }^{14,19}$ as the most common etiology of cellulitis in the community and in NHs is Streptococcus, not Staphylococcus. ${ }^{19,20}$ Other agents approved as first-line therapy in penicillin-allergic patients, such as clindamycin, ${ }^{14}$ were also prescribed less often than doxycycline for cellulitis. Further, the duration of therapy tended to be longer than recommended. Mild cellulitis, defined as erythema, warmth, and swelling, without fever or other signs of a systemic infection, should be treated with 5 days of antibiotics $^{14}$; however, in our data, the mean treatment course for cellulitis was 9.3 days, despite the fact that only one patient had a documented fever, which potentially indicated a more severe infection.

Abscesses were frequently diagnosed and treated in our sample. Again, doxycycline was the drug most commonly prescribed. However, the IDSA reserves doxycycline for severe abscesses in patients with systemic illness, or unsuccessful incision and drainage procedures. ${ }^{14}$ Our chart audits did not capture if an incision and drainage had been performed, but even if it had been, correct abscess management can be complex. Even dermatologists struggle with proper management of this frequent SSTI ${ }^{21}$ and often provide unrecommended oral therapy. In our cases, the lack of associated signs of systemic infection (eg, fever, leukocytosis) suggests that few residents were systemically ill from their abscesses.

The potentially overzealous prescribing practices in our NH sample may reflect the prescribers' aversion to the risks of delayed treatment given that $\mathrm{NH}$ residents carry a higher burden of sepsis than the general population. ${ }^{22}$ The overuse of doxycycline, both in indication and therapy duration, may reflect concern for MDROs such as methicillin-resistant $S$. aureus. Targeted educational interventions are advised to address these points; they should recognize the preconceptions and priorities of $\mathrm{NH}$ providers and engage the entire $\mathrm{NH}$ care team. ${ }^{13,23-26}$ Such opportunities can potentially improve antibiotic stewardship uniquely for SSTIs.

Previous studies have documented that nearly half of all $\mathrm{NH}$ antibiotic prescriptions were written by prescribers who were not physically present and therefore consulted by telephone, ${ }^{27}$ a situation that likely applies to our sample as well. An SSTI diagnosis requires a thorough examination, ${ }^{28}$ and accurate diagnoses can be achieved by better integrating technology with patient care. ${ }^{29}$ We never encountered documentation of a digital image used in the diagnostic process, but digital imagery can both enhance the visual aspects of a diagnosis and track symptom progression or resolution. Such systems already exist in the area of wound care, where some specialists use customized electronic health records to manage chronic wounds. ${ }^{30}$ Data suggest that health care information technologies promote better management decisions, ${ }^{30,31}$ and photographic documentation in NHs could potentially improve treatment choices and antibiotic stewardship for SSTI management.

\section{Conclusion}

From our sample of treated SSTIs, we conclude that the recommended standard of care is not practiced regularly. The McGeer criteria were created for surveillance purposes, the Loeb minimum criteria were generated to guide antibiotic initiation, and the IDSA recommendations were made to guide prescribing. None of these valuable resources appear to be used regularly in the $\mathrm{NH}$ setting to manage SSTIs. In the era of antibiotic stewardship, $\mathrm{NH}$ care may be improved and unnecessary antibiotic burden lessened by broader application of these guidelines. In addition, SSTI management may be improved by merging these resources with information technology. Just as MDROs are constantly evolving, so too must care in the $\mathrm{NH}$.

\section{References}

1. Trick W, Weinstein R, DeMarais P, et al. Colonization of skilled-care facility residents with antimicrobial-resistant pathogens. J Am Geriatr Soc 2001;49:270-276.

2. Fisch J, Lansing B, Wang L, et al. New acquisition of antibiotic-resistant organisms in skilled nursing facilities. J Clin Microbiol 2012;50:1698-1703.

3. US Centers for Disease Control and Prevention. The core elements of antibiotic stewardship in nursing homes. Available at: http://www.cdc.gov/ longtermcare/prevention/antibiotic-stewardship.html. Accessed June 30, 2016.

4. Federal Register: Medicare and Medicaid Programs; Reform of Requirements for Long-Term Care Facilities. Available at: https://www.federalregister.gov/ articles/2015/07/16/2015-17207/medicare-and-medicaid-programs-reformof-requirements-for-long-term-care-facilities\#h-12. Accessed June 30, 2016.

5. Dwyer L, Harris-Kojetin L, Valverde R, et al. Infections in long-term care populations in the United States. J Am Geriatr Soc 2013;61:341-349.

6. Beck-Sague C, Villarino E, Giuliano D, et al. Infectious diseases and death among nursing home residents: Results of surveillance in 13 nursing homes. Infect Control Hosp Epidemiol 1994;15:494-496.

7. Coleman E, Martau J, Lin M, Kramer A. Pressure ulcer revalence in long-term nursing home residents since the implementation of OBRA '87. J Am Geriatr Soc 2002;50:728-732.

8. Sibbald R, Woo K, Ayello E. Increased bacterial burden and infection: The story of NERDS and STONES. Adv Skin Wound Care 2006;19:447-461.

9. Garibaldi RA. Residential care and the elderly: The burden of infection. J Hosp Infect; 1999:S9-S18.

10. Loeb M, Bentley D, Bradley S, et al. Development of minimum criteria for the initiation of antibiotics in residents of long-term care facilities: Results of a consensus conference. Infect Control Hospital Epidemiol 2001;22:120-124. 
11. Olsho L, Bertrand R, Edwards E, et al. Does adherence to the Loeb minimum criteria reduce antibiotic prescribing rates in nursing homes? J Am Med Dir Assoc; 2013:309.e1-309.e7.

12. McGeer A, Campbell B, Emori T, et al. Definitions of infection for surveillance in long-term care facilities. Am J Infect Control 1991;19:1-7.

13. Stone N, Ashraf M, Calder J, et al. Surveillance definitions of infections in long term care facilities: Revisiting the McGeer criteria. Infect Control Hospital Epidemiol 2012;33:965-977.

14. Stevens D, Bisno A, Chambers H, et al. Practice guidelines for the diagnosis and management of skin and soft tissue infections: 2014 update by the Infectious Diseases Society of America. Clin Infect Dis 2014;59:147-159.

15. ICD-9 Code Lookup. Centers for Medicare \& Medicaid Services. Available at: https://www.cms.gov/medicare-coverage-database/staticpages/icd-9-code-look up.aspx? KeyWord $=709.9 \& \mathrm{bc}=$ AAAAAAAAAAAEAA\%3d\%3d\& Accessed June 30, 2016.

16. Blahd W. Fever temperatures: Accuracy and comparison topic overview. Available at: http://www.webmd.com/children/tc/fever-temperatures-accu racy-and-comparison-topic-overview. Accessed June 30, 2016.

17. Medicare.gov Nursing Home Compare. Centers for Medicare \& Medicaid Services. Available at: https://www.medicare.gov/nursinghomecompare/search. html. Accessed June 30, 2016

18. Nicolle L, Bentley D, Garibaldi R, et al. Antimicrobial use in long-term care facilities. Infect Control Hosp Epidemiol 1996;17:119-128.

19. Edwards M, Baker C, Group B. Streptococcal infections in elderly adults. Clin Infect Dis 2005;41:839-847.

20. Smith P, Bennett G, Bradley S, et al. SHEA/APIC Guideline: Infection prevention and control in the long-term care facility. Infect Control Hosp Epidemiol 2008; 29:785-814.
21. Chouake J, Krausz A, Adler B, et al. Management of cutaneous abscesses by dermatologists. J Drugs Dermatol 2014 Feb;13:119-124.

22. Ginde A, Moss M, Shapiro N, Schwartz R. Impact of older age and nursing home residence on clinical outcomes of U.S. emergency department visits for severe sepsis. J Crit Care 2013;28:606-611.

23. Rhee S, Stone N. Antimicrobial stewardship in long-term care facilities InfectDis Clin N Am 2014:28:237-246.

24. Dyar O, Pagani L, Pulcini C. Strategies and challenges of antimicrobial stewardship in long-term care facilities. Clin Microbiol Infect 2015;21:10-19.

25. Zimmerman S, Sloane P, Bertrand R, et al. Successfully reducing antibiotic prescribing in nursing homes. J Am Geriatr Soc 2014;62:907-912.

26. Scales K, Zimmerman S, Reed D, et al. Nurse and medical provider perspectives on antibiotic stewardship in nursing homes. J Am Geriatr Soc 2017;65 $165-171$.

27. Warren J, Palumbo F, Fitterman L, Speedie S. Incidence and characteristics of antibiotic use in aged nursing home patients. J Am Geriatr Soc 1991;39: 963-972.

28. Reddy M, Gill S, Wu W, et al. Does this patient have an infection of a chronic wound? JAMA 2012;307:605-611.

29. Rennert R, Golinko M, Kaplan D, et al. Standardization of wound photography using the wound electronic medical record. Adv Skin Wound Care 2008;22: 32-38.

30. Chaudhry B, Wang J, Wu S, et al. Systematic review: Impact of health information technology on quality, efficiency, and costs of medical care. Ann Intern Med 2006;144:742-752.

31. Ammenwerth E, Schnell-Inderst P, Machan C, Siebert U. The effect of electronic prescribing on medication errors and adverse drug events: A systematic review. J Am Med Inform Assoc 2008;15:585-600. 